\title{
PULMONARY TUBERCULOSIS AMONG PREGNANT MOTHERS IN TANZANIA
}

Authors:

Sheriff FG (MD), Manji KP (MD, MMED, MPH), Manji MP (MD), Chagani MM (MD)

(All affiliated to Muhimbili University of Health and Allied Sciences (MUHAS), Tanzania)

\section{ABSTRACT}

\section{OBJECTIVES}

Determining the prevalence of Pulmonary Tuberculosis (PTB) during pregnancy in Northern Tanzania.

\section{DESIGN}

In a prospective cross-sectional study.

\section{RESULTS}

Latent Tuberculosis Infection (LTBI) was detected in 86 out of 287 pregnant women (29.9\%). Active PTB was diagnosed radiologically in four subjects (1.4\%). All cases were sputum smear-negative

\section{CONCLUSION}

LTBI and subsequently active PTB is a public health problem among pregnant mothers in Northern Tanzania.

\section{INTRODUCTION}

It is estimated that TB infection is present in onethird of the world's population, or two billion people. Eight million new cases of active disease have been estimated to occur worldwide annually. ${ }^{1}$ Currently, more than 3 million people die from tuberculosis every year. The greatest burden of latent TB infection is found in Southeast Asia (prevalence, 46\%), the Western Pacific region (32\%), Africa (31\%), and the Eastern Mediterranean region (27\%). ${ }^{2}$

Women between the ages of 15 and 49 years carry the greatest risk of converting from tuberculosis infection to the disease. ${ }^{3}$ It is expected that the incidence of tuberculosis among pregnant women would be as high as in general population. In a South African study, TB was the third leading cause of maternal mortality, mostly in combination with HIV-infection. ${ }^{4}$ Neonatal mortality and extreme prematurity have also increased significantly due to the problem. ${ }^{5}$ Tanzania has not been spared. ${ }^{6}$ In spite of these high figures, reports of this combined infection in pregnancy have been sparse. ${ }^{7}$

The study was aimed at determining the prevalence of PTB in pregnancy and co-infection with HIV in Tanzania.

\section{METHODOLOGY}

\section{Settings}

An urban hospital based cross-sectional study was undertaken at the Ngarenaro and Levelosi clinics. These are resource poor settings with minimal microbiological lab support. The study population included all consenting pregnant women attending clinic during a total study duration of 4 weeks, with an intention to treat. Allowing for a $20 \%$ failure to follow up, a minimum sample size of 395 was reached. Ethical clearance was obtained from the MUHAS community health department and the National Tuberculosis and Leprosy Program.

\section{Screening Methods}

Eligible participants in our study were subjected to a TB symptom check-list followed by a thorough physical examination. They were also subjected to the Mantoux test which involved injection of $0.1 \mathrm{ml}$ of PPD (Purified Protein Derivative) RT 23 SSI intradermally using standard procedures. Results were interpreted 48 hours later using the ball-point pen method $^{8}$ and proof read by a senior researcher. Those with a productive cough or cough for at least 2 weeks had 
their "spot" and "morning" sputum screened for Acid Fast Bacilli (AFB).

Sputum specimens were dissolved in an equal volume of household bleach ( $5 \%$ Sodium Hypochlorite), then left for 10 minutes at room temperature $\left(25^{\circ} \mathrm{C}\right)$ with vigorous shaking at regular intervals. This was followed by addition of $8 \mathrm{ml}$ of distilled water after which the suspension was centrifuged at $3000 \mathrm{~g}$ 's for a further $10 \mathrm{~min}$. The supernatant was carefully poured off, and the sediment stained using Ziehl Nielsen's technique. This centrifugation technique has been shown to be superior to direct microscopy as far Acid fast bacterial yield (sensitivity) is concerned. ${ }^{9}$ The slides were interpreted by two independent laboratory technicians in a blinded fashion.

All those with productive cough, cough lasting more than two weeks or a positive Tuberculin Skin Test (TST) were subjected to a single postero-anterior chest radiograph (CXR) with abdominal shielding. The CXRs were interpreted by two independent radiologists in a blinded fashion. Pregnant mothers found to be having Active Pulmonary Tuberculosis were counselled and referred to a TB centre for treatment and follow-up.

\section{Data processing and analysis}

The data was processed using the Epi-Info v. 6 statistical package. Fischer's exact p-values, Chi-squares, regression analyses and Odd's ratios were calculated. A Fisher's exact 2-tailed P-value of less than 0.05 was considered significant. Scatter plots were utilized in the detection of trends and patterns.

\section{Inclusion criteria}

Pregnant women with gestational ages from 12 weeks to full term were included in the study.

\section{RESULTS}

Of the 396 women initially screened, 287 (72.5\%) returned to have their Tuberculin Skin Tests (TSTs) read. The mean age of those screened was 24.9 years, ranging from 16 to 40 years. The mean Body Mass Index for this subpopulation stood at $25.06 \mathrm{~kg} / \mathrm{m}^{2}$. $\left(95 \% \mathrm{Cl}=24.6-25.5 \mathrm{~kg} / \mathrm{m}^{2}\right)$

The mean TST induration in those with a positive result was $19.4 \mathrm{~mm}$ ( $95 \% \mathrm{Cl} 18.2-20.5 \mathrm{~mm})$. A positive TST result was defined as at least $10 \mathrm{~mm}$ induration for HIV negative patients and $5 \mathrm{~mm}$ for HIV positive ones.

Table 1: Prevalence of Latent TB infection, Active PTB and HIV among pregnant women attending ANC

\begin{tabular}{|l|l|l|}
\hline TST INTERPRETATION (LTBI) & Frequency & Percent \\
\hline POSITIVE & 86 & $29.9 \%$ \\
\hline NEGATIVE & 201 & $70.1 \%$ \\
\hline TOTAL & 287 & $100.0 \%$ \\
\hline PTB (Dx by CXR) & Frequency & Percent \\
\hline NO & 283 & $96.6 \%$ \\
\hline OLD & 6 & $2.0 \%$ \\
\hline ACTIVE & 4 & $1.4 \%$ \\
\hline TOTAL & 293 & $100.0 \%$ \\
\hline HIV sero status & Frequency & Percent \\
\hline Positive & 20 & $5.1 \%$ \\
\hline Negative & 376 & $94.9 \%$ \\
\hline TOTAL & 396 & $100.0 \%$ \\
\hline
\end{tabular}

A weak positive trend $(P>0.05)$ of increasing proportion of positive TSTs with age was noticed which was more pronounced after the age of 30 . No significant correlation was observed between $\mathrm{LTBI}$ (latent TB infection) and marital status, parity or gestational age. HIV infection influenced TST conversion inversely; the TST positive rate among HIV positive women (23.1\%) being lower than that of their HIV negative counterparts $(30.3 \%)$

A history positive for 'other contact' of open PTB in the previous two years appeared to confer an increased risk for developing lesions suggestive of active PTB on CXR (OR=16.0; 95\% Cl(1.5,175.6); $\mathrm{P}<0.05)$. 'Other contact' referred to one outside the nuclear family but limited to the immediate neighborhood or workplace - it included extended family. When statistical analysis for the combined risk conferred by "family" AND/OR "other" TB contacts was carried out, the following results were obtained: Odd's Ratio $=8.6 ; 95 \% \mathrm{Cl}(0.8$ $<\mathrm{OR}<89.9$ ); $\mathrm{P}=0.06$.

Of the symptoms reported by pregnant women in our study the most common were malaise (36.6\%), loss of appetite $(19.2 \%)$, pleurisy $(14.1 \%)$ and fever (13.1\%) (Table 2). Most (88.4\%) women had no abnormalities on percussion or auscultation. Among the four active PTB subjects, anomalies detected on examination included a pleural 
effusion and rhonchi in one (1) case each. The latter feature i.e. rhonchi was reported in an HIV/ PTB co-infected subject. Only two symptoms showed a strong positive correlation with a chest $X$-ray diagnosis of active PTB. These were cough for more than two weeks (OR=63.7; 95\% Cl (5.2, 1741.7); $P<0.01)$ and productive cough $(O R=42.6$; $95 \% \mathrm{Cl}(3.6,1138.9) ; \mathrm{P}<0.01)$. One $(25 \%)$ of the four active PTB cases diagnosed was completely asymptomatic.

Table 2: Frequency distribution of clinical features suggestive of PTB among all pregnant women:

\begin{tabular}{|l|l|l|}
\hline Clinical features (symptoms) & Frequency & $\begin{array}{l}\text { Percent } \\
\text { (of 396) }\end{array}$ \\
\hline Malaise & 145 & $36.6 \%$ \\
\hline Loss of Appetite & 76 & $19.2 \%$ \\
\hline Pleurisy & 56 & $14.1 \%$ \\
\hline Fever & 52 & $13.1 \%$ \\
\hline Dyspnoea & 33 & $8.3 \%$ \\
\hline Weight Loss & 30 & $7.6 \%$ \\
\hline Productive Cough & 26 & $6.6 \%$ \\
\hline Cough > 2 weeks & 20 & $5.1 \%$ \\
\hline Night Sweats & 18 & $4.5 \%$ \\
\hline Haemoptysis & 1 & $0.3 \%$ \\
\hline Clear & 350 & $88.4 \%$ \\
\hline Rhonchi & 21 & $5.3 \%$ \\
\hline Crackles & 16 & $4.0 \%$ \\
\hline Bronchial breath sounds & 8 & $2.0 \%$ \\
\hline Pleural rub & 1 & $0.3 \%$ \\
\hline Pleural effusion & 1 & $0.3 \%$ \\
\hline
\end{tabular}

Radiologic changes consistent with a diagnosis of active PTB were present in a total of four patients, two of whom were HIV co-infected. Radiologic findings which were significantly associated with active PTB included hilar shadowing (OR 30.3, $95 \% \mathrm{Cl}$ 2.37-852.4, $\mathrm{P}<0.01$ ); cavitation especially apical $(\mathrm{OR}=110 ; 95 \% \mathrm{Cl} 4.6-5714, \mathrm{P}<0.01)$, pleural effusion, atelectasis and hilar lymphadenopathy (all with OR undefined, $\mathrm{P}<0.05$ ).

Out of 28 subjects whose sputum was obtained for examination, no AFB positive smears were detected. Hence all cases diagnosed based on chest radiography were smear negative for Pulmonary tuberculosis.

\section{DISCUSSION}

The prevalence of Latent tuborculosis infection (LTBI) among pregnant women in this study was $29.9 \%$ and does not differ significantly from that of other African countries (31\%). ${ }^{11}$ Acquisition of TB infection is highest during reproductive age and pregnancy does not alter the immunological reactivity to tuberculin as indicated in several studies. ${ }^{12,13}$ Active PTB in this population $(1.4 \%)$ is quite prevalent considering the range of 0.1\%-1.9\% derived by two independent researchers, Hamadeh and Tran JH. ${ }^{14,15}$ This is worrying considering the fact that in Sub-Saharan Africa, TB is becoming an increasingly important cause of non-obstetric mortality ${ }^{4}$ and adverse pregnancy outcomes. ${ }^{16}$ According to the Tanzania Demographic and Health Survey (TDHS) 2004/05, the prevalence of pregnancy among a subpopulation of 15-49 year old females was $10.5 \%$. Considering the active PTB prevalence from our study $1.4 \%$ gives an alarming estimate of 12,121 cases of active PTB in pregnancy, nationwide, at any given point in time. ${ }^{17}$ Furthermore, the risk of motherto -child-transmission for active disease is $15 \%$ within 3 weeks of delivery. ${ }^{18}$

The relationship between age and TST results may be the effect of cumulative exposure to $M$. tuberculosis over several years, since an individual's risk of exposure is a product of concentration of infected droplets in the air and the length of time a person breathes that air. ${ }^{21}$ We found that parity had no significant influence on the incidence of PTB, a finding echoed by a South African study which revealed no significant difference between those pregnant women diagnosed with PTB and those who were not as far as age and parity were concerned. ( $\mathrm{P}=0.9,0.8$ respectively). ${ }^{4}$

No cases of active PTB had a history of 'family contact' in our study, but half of the diagnosed active PTB cases had a history of 'other contact' exposure within the preceeding two years $(\mathrm{OR}=16.0, \mathrm{P}<0.05)$. These results are similar to those in condcuted among a study in Iraqi children, which showed that those living in extended families had a higher TB infection rate compared to those living within nuclear families $(r=0.72, P<0.05) .{ }^{22}$

Previous studies including a prominent one published by Centre for Decease Control, Atlanta have reported that HIV positive subjects are less likely to be 
TST-positive than HIV-negative ones; something that we also noted. ${ }^{23}$ This is likely due to the defective cellmediated immune response in this high risk group which is necessary for delayed type hypersensitivity, the basis of the tuberculin skin test. Another limitation of tuberculin skin testing brought to light in our study was its low Positive Predictive Value (2.4\%) for active PTB making it economically and logistically unsuitable for mass screening. This is the reason why many authorities now recommend its use be limited to screening among high riskindividuals, chiefly consisting of patients suffering from immunosuppression and chronic debilitating diseases..$^{24.25}$

The two most common symptoms reported by pregnant women in our study were malaise $36.6 \%$ and loss of appetite $19.2 \%$ and other symptoms including dyspnoea (8.3\%) were likely due to the physiological changes of pregnancy itself, which often mimic the earlier constitutional symptoms of PTB. ${ }^{29}$ The most common symptoms reported in a South-African study by Kali et al were malaise, cough, productive cough and shortness of breath. ${ }^{26}$ As far as HIV positive pregnant women are concerned; the occurrence of constitutional symptoms further increases the diagnostic difficulty as they can be caused by HIV infection itself.

Radiology is highly sensitive for PTB as demonstrated by Good et al in whose study all the 27 pregnancies diagnosed with culture positive PTB had abnormal $\mathrm{CXRs}^{27}$, this can further be improved when combined with symptom screening. The CXR findings reported in the cases of active PTB without HIV co-infection were hilar shadows and apical cavitation (both cases), right sided pleural effusion and infiltrates in the mid and upper zones (one case). HIV positive subjects with active PTB had atypical patterns on CXR: one case had infiltrates in the Left upper zone with atelectasis as shown by elevation of the left hemi diaphragm. One of the patient had been treated for PTB three years prior to our study, but was showing clinical features of disease reactivation which was confirmed radiologically. Another co-infected case had micro nodules $(>3 \mathrm{~mm})$ in the lower zone and hilar lymph node enlargement with calcification. No cavitation or pleural effusions were noticed in the HIV positive group. In the later stages of HIV infection, the CXR appearance of active PTB resembles that of primary
TB often with infiltrates rather than cavities. Also, the lower zones are more likely to be involved than the upper ones.

Our study revealed two symptoms having a positive correlation with CXR diagnosis of PTB: cough for more than two weeks (Odd's ratio(OR) 63.7) and productive cough $(O R, 42.6)$. One of the four women diagnosed with active PTB in our study was asymptomatic. Comparing our study with that of Kali et al , clinical features appearing with similar frequencies in both studies included cough (75\% vs. $74 \%$ ), fever (25\% vs. $30 \%$ ) and asymptomatic ( $25 \%$ vs. $20 \%$ ). On chest examination, abnormalities were detected in only two of the four active PTB cases, pleural effusion in one case and scattered rhonchi over the posterior upper thorax in the other. It is known that the physical exam in PTB may reveal scattered rhonchi and wheezes, 18 however a history of atopy is usually against a diagnosis of PTB. In fact, a finding of crackles actually decreases the likelihood of a CXR diagnosis of PTB. ${ }^{10}$

All sputum smears in our study were AFB negative; a factor associated with lower risk of mother-tochild transmission (MTCT) of TB. Our findings are not isolated since a study at Rhodes island, 1993, showed pregnant women with pulmonary conditions were more likely to present with unilateral non-cavitary, smear-negative disease $(p=0.02){ }^{28}$ Similarly, Kali et al in a South African study, found that all eight pregnant women diagnosed with active PTB were sputum AFB negative but culture positive. ${ }^{26}$

The risk score derived in this study is of particular value in pregnant women situated in low resource settings since it uses no lab/radiographic parameters. As compared to the risk score derived by Wisnivesky, it has a higher PPV (Positive Predictive Value) and Specificity, but a lower Sensitivity. Our risk score may also be useful as a tool for excluding active PTB before initiation of Isoniazid prophylactic therapy(IPT) and for screening HIV positive candidates prior to vaccination with the newly developed M.vaccae vaccine..$^{29}$ In any case, well structured case control studies are required to verify its statistical significance. 


\section{CONCLUSION}

The prevalence of latent TB, active PTB and co infection with HIV among pregnant women in Tanzania is relatively high with direct implications on the incidence of neonatal tuberculosis. These findings necessitate more serious thought on the issue of targeted TB screening during pregnancy keeping in mind the consequences of late diagnosis, the nonspecific presentation of the disease during pregnancy and the specific needs and vulnerabilities of both mother and fetus. Once found to have a positive TST in the absence of laboratory radiographic evidence for active disease, the issue of using Isoniazid Prophylactic Therapy (IPT) comes to surface and needs to be clearly addressed in standard guidelines.

\section{ACKNOWLEDGEMENTS}

Dr. M Pallangyo, Dr. J Lyimo (Radiologist), Dr. A Jusabani (Radiologist and Lecturer-KCMC referral hospital), Dr. L Kaaya, Dr. Z Dosaji, T Shabbir T, Z Alwani, MK Alwani and D Silveira.

\section{REFERENCES}

1. World Health Organization (WHO). Global tuberculosis control surveillance, planning, financing: WHO report 2006. WHO/HTM/TB/2006.362. Geneva: WHO, 2006.

2. Corbett EL, Watt CJ, Walker N, et al. The growing burden of tuberculosis: global trends and interactions with the HIV epidemic. Arch Intern Med 2003;163:1009-21.

3. Holmes $C B$, Hausler $H$, Nunn P. A review of sex differences in the epidemiology of tuberculosis. Int J Tuberc Lung Dis 1998; 2: 96-104.

4. Khan M, Pillay T, Moodley JM, Connolly CA. Maternal mortality associated with tuberculosis-HIV-1 coinfection in Durban, South Africa. AIDS 2001,15: 1857-63.

5. Tripathy S. N., Tripathy S. N. Tuberculosis and pregnancy. Int J Gynaecol Obstet 2003; 80(3):247-53.

6. National Aids Control Programme (NACP) HIV/AIDS/STD Surveillance. Ministry of Health and Social Welfare Tanzania 1998; Report No. 13.

7. Margono F, Mroueh J, Garely A et al. Resurgence of active tuberculosis among pregnant women. Obstet Gynecol 1994, 83: 911-914.

8. Harriman CD and Cohen Fl. Screening for tuberculosis: an important prevention tool. In: Cohen Fl and Durham JD. Eds. Tuberculosis: A Sourcebook for Nursing Practice. New York, New York: A Springer Publishing Company, 1995: 139-155.

9. Steingart $K R, N g V$, Henry $M$ et al. Sputum processing methods to improve sensitivity of smear microscopy for tuberculosis: a systematic review. Lancet Infect Dis. 2006 Oct; 6(10): 664-74

10. Wisnivesky JP, Kaplan J, Henschke $C$ et al. Evaluation of clinical parameters to predict Mycobacterium tuberculosis in Inpatients. Arch. Internal Med. 2000; 160: 2471-2476.

11. Corbett EL, Watt CJ, Walker N, et al. The growing burden of tuberculosis: global trends and interactions with the HIV epidemic. Arch Intern Med 2003; 163:1009-21.

12. Khilnani, G.C. Tuberculosis and Pregnancy. Indian J Chest Dis Allied Sci 2004; $46: 105-111$.

13. Pridie RB, Stradling P. Management of pulmonary tuberculosis during pregnancy. BMJ 1961;3: 78-81.

14. Hamadeh MA, Glassroth J. Tuberculosis and pregnancy. Chest 1992; 101:1114-20.

15. Tran JH, Montakantikul P. The safety of antituberculosis medications during breastfeeding. J Hum. Lactation 1998; 14:337-40. 
16. Jana N, Vasishta K, Jindal SK, et al. Perinatal outcome in pregnancies complicated by pulmonary tuberculosis. Int J Gynecol Obstet 1994; 44:119-124

17.Tanzania Demographic and Health Survey, 2004/05: http://www.nbs.go.tz/library.htm [cited $4^{\text {th }}$ Nov 2008]

18. Pillay $T$, Khan M, Moodley $J$ et al. Perinatal tuberculosis and HIV-1: considerations for resourcelimited settings. Lancet Infect Dis 2004; 4: 155-65

19. World Health Organization (WHO). Global tuberculosis control surveillance, planning, financing: WHO report 2006. WHO/HTM/TB/2006.362. Geneva: WHO, 2006.

20. National Tuberculosis and Leprosy Program Manual - Ministry of Health and Social Welfare Tanzania 2006; p 51. Available from website of MoHSW: http://www.moh.go.tz/NTLP/documents. php [Last accessed: 16th Nov 2008]

21. National Tuberculosis and Leprosy Program Manual - Ministry of Health and Social Welfare Tanzania 2006; p 6-7 Available from website of MoHSW: http://www.moh.go.tz/NTLP/documents. php [Last accessed: 16th Nov 2008]

22. Kubaisy W., Dulayme A, Hashim DS. Active tuberculosis among Iraqi schoolchildren with positive skin tests and their household contacts. East Mediterr Health J. 2003; 9(4):675-88.

23. Centers for Disease Control and Prevention. Missed Opportunities for Prevention of Tuberculosis among Persons With HIV Infection --- Selected Locations, United States, 1996-1997. MMWR: 49(30); 685-7.

24. Centers for Disease Control and Prevention. Targeted tuberculin testing and treatment of latent tuberculosis infection. MMWR [Internet] 2000; 49/No.RR-6:1-51. [cited February 15, 2005]. Available from: $h$ ttp://www.cdc.gov/mmwr/preview/mmwrhtml/rr4906a1.htm.

25. Gillum MD, Maki DG. Tuberculin testing, BCG in pregnancy. Infect Control Hosp Epidemiol 1988;9:119-2126. Kali P, Gray G. PMTCT With Active Case Finding for Tuberculosis. Epidemiology and Social Science report 2006; 42(3):379-381

27. Good JT, Iseman MD, Davidson PT, et al. Tuberculosis in association with pregnancy. Am J Obstet Gynecol 1981;140:492-498

28. Carter E.J, Mates S. Tuberculosis During Pregnancy: The Rhode Island Experience, 1987 to 1991.Chest 1994;106:1466-1470

29. http://www.scidev.net/en/news/vaccine-slashes-tb-infection-in-hiv-patients.html [cited 29th Oct 2008] 